\title{
Psychological Impacts Related to Stress and Fear during the COVID-19 Pandemic: Cardiovascular Diseases, Diabetes and Psychological Disorders as Risk Factors
}

\author{
Lucas Emmanuel Pedro de Paiva Teixeira ${ }^{1^{*}}$ (1), Renato Leonardo de Freitas ${ }^{2,3,4}$ (),

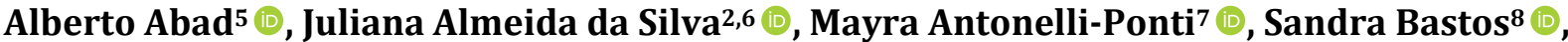 \\ Cláudia Helena Cerqueira Mármora9 ${ }^{\circledR}$, Luis Antonio Monteiro Campos ${ }^{10,11}$ (1), \\ Scheila Paiva ${ }^{5,12}$ (), José Aparecido Da Silva13 ${ }^{10}$ \\ ${ }^{1}$ Institute of Motricity Sciences, Federal University of Alfenas (UNIFAL-MG), Alfenas, Brazil \\ ${ }^{2}$ Behavioural Neurosciences Institute (INeC), Ribeirão Preto, Brazil \\ ${ }^{3}$ Biomedical Sciences Institute, Federal University of Alfenas (UNIFAL), Alfenas, Brazil \\ ${ }^{4}$ Laboratory of Neurosciences of Pain \& Emotions and Multi-User Centre of Neuroelectrophysiology, Department of Surgery and \\ Anatomy, Ribeirão Preto Medical School of the University of São Paulo, Ribeirão Preto, Brazil \\ ${ }^{5}$ Psychology Graduate Program, Federal University of Juiz de Fora (UFJF), Juiz de Fora, Brazil \\ ${ }^{6}$ Laboratory of Neuroanatomy and Neuropsychobiology, Department of Pharmacology, Ribeirão Preto Medical School of the \\ University of São Paulo (FMRP-USP), Ribeirão Preto, Brazil \\ ${ }^{7}$ Laboratory of Studies and Research in Social Economy, University of São Paulo at Ribeirão Preto, Ribeirão Preto, Brazil \\ ${ }^{8}$ Otorhinolaryngology Institute (ISBO), São Paulo, Brazil \\ ${ }^{9}$ Department of the Old, Adult and Maternal-Infant, School of Physical Therapy, Graduate Program in Psychology, Federal \\ University of Juiz de Fora (UFJF), Juiz de Fora, Brazil \\ ${ }^{10}$ Master's Program in Psychology, Catholic University of Petrópolis, Petrópolis, Brazil. \\ ${ }^{11}$ Department of Psychology, Pontifical Catholic University of Rio de Janeiro, Rio de Janeiro, Brazil \\ ${ }^{12}$ Department Speech Therapy of Federal University of Sergipe (UFS), Lagarto, Brazil \\ ${ }^{13}$ Laboratory of Psychophysics, Perception, Psychometrics, and Pain, University of São Paulo at Ribeirão Preto, \\ Ribeirão Preto, Brazil \\ Email: *lucas.teixeira@unifal-mg.edu.br
}

How to cite this paper: Teixeira, L.E.P.P., de Freitas, R.L., Abad, A., da Silva, J.A., Antonelli-Ponti, M., Bastos, S., Mármora, C.H.C., Campos, L.A.M., Paiva, S. and Da Silva, J.A. (2020) Psychological Impacts Related to Stress and Fear during the COVID19 Pandemic: Cardiovascular Diseases, Diabetes and Psychological Disorders as Risk Factors. World Journal of Neuroscience, 10, 191-205.

https://doi.org/10.4236/wins.2020.104019

\begin{abstract}
Background and Aims: Patients and the general public are under insurmountable psychological pressure which may lead to various psychological problems, such as anxiety, fear, depression, and insomnia, causing, consequently, the impaired quality of life. Psychological crisis intervention plays a pivotal role in the overall deployment of health-related quality of life and disease control. A novel Severe Acute Respiratory Syndrome Coronavirus-2 (SARSCoV-2), a pathogen of the new coronavirus disease (COVID-19), has affected several sector activities, including people's health. To enhance infection con-
\end{abstract}


Received: September 16, 2020

Accepted: November 6, 2020

Published: November 9, 2020

Copyright () 2020 by author(s) and Scientific Research Publishing Inc. This work is licensed under the Creative Commons Attribution International License (CC BY 4.0).

http://creativecommons.org/licenses/by/4.0/ trol methods, appropriate interventions, and public health policies, the present study aims to assess the fear and peri-traumatic stress during the COVID-19 in Brazil. Method: A cross-sectional survey has been conducted from April $12^{\text {th }}$ to $18^{\text {th }}$ from 2020, using the Peri-Traumatic Distress Scale (CPDI) and the Fear Scale (FCV-19S) aiming to measure the peri-traumatic stress and fear as psychological reactions during the COVID-19 pandemic. For that purpose, an online spreadsheet was used to send the questionnaire and scales to a sample of 1844 participants as a collecting information tool. After the data analysis, the individuals were separated into 4 groups: Group 1 (1232) population without chronic health conditions; group 2 (298) patients with previous psychological suffering, group 3 (229) patients with cardiovascular diseases, group 4 (71) patients with diabetes. For analysis, G1 were considered control for comparison with groups 2, 3 and 4. Results: All the groups showed the CPDI and FCV-19S increased in comparison with the G1 group. Concerning CPDI, the G 3 was increased when compared to G1, G2 and G4. The G3 had the FCV-19S higher in comparison with G1, G2 and G4. The Wilcoxon-Mann-Whitney test showed a statistical difference between the control group in comparison with 2 and 3 groups (Mann-Whitney $\mathrm{p}<0.05$ ). Conclusion: The COVID-19 pandemic had a significant impact on the Brazilian population, with patients with heart disease and hypertension presenting the highest numbers of stress and fear, with numbers comparable and even higher than those who reported previous psychological distress.

\section{Keywords}

Mental Health, Anxiety Reactions, Fear, Peri-Traumatic Stress, Cardiovascular Diseases, Diabetes, COVID-19, Pandemic

\section{Introduction}

The COVID-19 pandemic of the new coronavirus (SARS-CoV-2) has presented itself as one of the greatest health challenges on a global scale this century. By mid-September, a few months after the outbreak in China in late 2019, there had been more than 29 million cases and 930,000 deaths worldwide from COVID-19.

Abad and colleagues (2020) evidenced that the COVID-19 started in China and took over the world becoming a pandemic, arriving in Latin America in mid-February 2020. The South American continent is going through a delicate political, economic, and social moment that reflects the fragility of our health systems and science, contagion rates reduction strategies, and health services overload. Nevertheless, several studies are being carried out in Brazil and worldwide aiming to understand the characteristics of the disease and the cure of people infected by the coronavirus. Therefore, we must consider science-based strategies to combat the pandemic and promote a health system that takes care of the population [1].

Finally, to control the viral spread in Brazil, it is of utmost importance to have 
a clear scientific-based pandemic control national guideline (risk-communication, hygiene, and social distance practices), the use of medications recommended by the World Health Organization (WHO) and health professionals on key positions in the Health Ministry.

No one can predict how things will evolve in the coming months, or when a return to some semblance of "normal" activity might resume [2], this can lead the population to experience stress, fear, anguish for a long time.

Previous research has revealed a profound and wide range of psychosocial impacts on people at the individual, community, and international levels during outbreaks of infection. On an individual level, people are likely to experience fear of falling sick or dying themselves, feelings of helplessness, and stigma [3].

Abad and collaborators (2020) studied the Peri-Traumatic Distress Scale (CPDI) and the Fear Scale (FCV-19S) aiming to measure the peri-traumatic stress and fear as psychological reactions during the Covid-19 pandemic. In fact, the CPDI and FCV-19S results show gender difference scores as a response to the pandemic. It is paramount that women's voices were represented in policy spaces as socially constructed gender roles place them in a strategic position to enhance multi-level interventions (primary and secondary effects of Covid-19), equitable policies, and new approaches to control the pandemic [4].

Pre-existing comorbidities such as hypertension, diabetes, and cardiovascular disease are associated with greater severity and higher fatality rate of COVID-19. Furthermore, COVID-19 contributes to cardiovascular complications, including acute myocardial injury as a result of acute coronary syndrome, myocarditis, stresscardiomyopathy, arrhythmias, cardiogenic shock, and cardiac arrest [5].

Depression and anxiety are psychiatric disorders that often coexist with coronary artery disease (CAD) and other cardiovascular diseases (CVD). Both depressive symptoms and anxiety are currently recognized as risk factors for CAD and CVD, and present complex pathophysiological processes that seem to adversely influence the prognosis of patients with these comorbidities. These symptoms include hypercortisolism, sympathetic hyperactivity, complex platelet abnormalities, immune activation leading to inflammatory response, common genetic factors and association with behaviours that predispose to cardiovascular disease [6].

In a systematic review that included six studies with a total of 1527 patients, $\mathrm{Li}$ et al., 2020, evaluated the proportions of hypertension, cardio-cerebrovascular disease and diabetes in patients with COVID-19. The incidences of hypertension, cardio-cerebrovascular disease and diabetes were two to three times and twice, respectively, higher in ICU/severe cases than in non-ICU/severe patients. At least $8.0 \%$ of patients with COVID-19 suffered an acute cardiac injury. The incidence of acute cardiac injury was about 13 times higher in ICU/severe patients compared to non-ICU/severe patients [7].

Studies show that individuals with severe illness or multiple comorbidities present higher levels of psychological symptoms in the face of this COVID-19 
pandemic situation. In a study that assessed the impact of the COVID-19 pandemic in Spain, Ozamiz-Etxebarria et al., 2020, showed that individuals who reported chronic diseases had higher average levels of stress, anxiety and depression compared to those who did not report such diseases [8].

Taking into consideration that hypertensive and diabetic patients are part of the group at risk for the severity of coronavirus infection and that research shows that people with chronic diseases have high levels of psychic suffering caused by COVID-19, it is necessary to evaluate the impact of the pandemic in patients with chronic diseases, especially cardiovascular and diabetes.

In short, in a pandemic of proportions never seen before in Brazil as the one we are now facing, it is important to investigate the psychological impact of the pandemic on real populations so that health authorities can develop strategies to reduce psychological symptoms in a targeted manner to the most vulnerable population. Thus, this study measured stress levels and fear in a sample of adults from all regions of Brazil during the pandemic by COVID-19, making it possible with the data collected to analyze the psychological needs of those facing the pandemic and the possible predictive factors.

\section{Methods}

A cross-sectional survey has been conducted from April $12^{\text {th }}$ to $18^{\text {th }}$ from 2020, using the Peri-Traumatic Distress Scale (CPDI) and the Fear Scale (FCV-19S) aiming to measure peri-traumatic stress and fear as psychological reactions during the Covid-19 pandemics. For that purpose, an online spreadsheet was used to send the questionnaire and scales to a sample of 1844 participants as a collecting information tool. The volunteers were selected by a non-probabilistic sample, through social networks, e-mail and messages via cell phone applications. After the data analysis, the individuals were separated into 4 groups: Group 1 (1232) population without chronic health conditions, group 2 (298) patients with previous psychological suffering, group 3 (229) patients with cardiovascular diseases, group 4 (71) patients with diabetes. For analysis, G1 were considered control for comparison with groups 2,3 and 4 .

Participants first answered a socio-demographic survey that included specific questions about chronic disease prevalence and social isolation levels during the COVID-19 pandemic. Then, we assessed distress by the Peri-Traumatic Distress Index (CPDI), Appendix 1, designed as a self-report questionnaire that measures depression, anxiety, avoidance, compulsive behaviour, specific phobias, cognitive change, physical symptoms, and loss of social functioning. The twentyfour questions were presented in a Likert format in five categories of responses (never, occasionally, sometimes, often, most of the time). CPDI content has been validated by Qiu et al., 2020 [9].

We measured fear with the COVID-19 Fear scale (FCV-19S), Appendix 2 , presented in a Likert format in five categories of responses (strongly disagree, disagree, neither agree nor disagree, agree, strongly agree). It consists of a sev- 
en-item unidimensional scale with robust psychometric properties reliable and valid in assessing and relieving fears of COVID-19 among individuals [10]. Both scales, FCV-19S and CPDI were authorized by the authors to be used in the research and were translated and adapted to the Brazilian Portuguese language.

Concerning the statistical analyses, the data are presented as median, $25^{\text {th }}$ to $75^{\text {th }}$ percentiles. First, the reliability of the two scales was evaluated using the Cronbach's Alpha calculation, Bartlett's sphericity test and the Kaiser-Meyer-Olkin adequacy factor (KMO). Then, the Shapiro-Wilk normality test was performed to check the normal distribution of all data. Data regarding the Fear scale and Peritraumatic distress were subjected to a one-way repeated-measures analysis of variance (split-plot ANOVA), followed, when appropriate, by Tukey's post hoc test.

This research was approved by the National Research Ethics CommissionCONEP, Brazil.

\section{Results}

First, it was showing the proportion of the interviewees who have health problems, who were in Social isolation, and who have physical disabilities and proportion who practice some sport activity and proportion by age group, as shown in Table 1.

Table 2 present the means, confidence intervals, weighted means and the

Table 1. Descriptive table of the interviewees' health data

\begin{tabular}{|c|c|c|c|}
\hline Variable & Classes & Proportion (\%) & $\begin{array}{l}\text { Proportion Confidence } \\
\text { Interval (IC)- } 95 \%\end{array}$ \\
\hline \multirow[t]{3}{*}{ With health problem } & No & 65.40 & $63.17-67.57$ \\
\hline & Yes & 34.60 & $32.43-36.83$ \\
\hline & TOTAL & 100.00 & - \\
\hline \multirow[t]{3}{*}{ Social isolation } & No & 12.96 & $11.48-14.60$ \\
\hline & Yes & 87.04 & $85.40-88.52$ \\
\hline & TOTAL & 100.00 & - \\
\hline \multirow[t]{3}{*}{ Physical disability } & No & 98.43 & $97.72-98.93$ \\
\hline & Yes & 1.57 & $1.07-2.28$ \\
\hline & TOTAL & 100.00 & - \\
\hline \multirow[t]{3}{*}{ Practices sports activity } & No & 52.01 & $49.70-54.31$ \\
\hline & Yes & 47.99 & $45.69-50.30$ \\
\hline & TOTAL & 100.00 & - \\
\hline \multirow[t]{4}{*}{ Age group } & Less than 25 & 21.20 & $19.37-23.16$ \\
\hline & $26-50$ years & 63.07 & $60.81-65.27$ \\
\hline & 51 or more & 15.73 & $14.11-17.49$ \\
\hline & TOTAL & 100.00 & - \\
\hline
\end{tabular}


Cronbach Alpha estimate for each of the factors of the respective CPDI scales and the Fear scale. The CPDI scale composed of 4 factors is represented there, as well as the Fear scale, which is unidimensional, in which we use the mean Fear Score for the exposed calculations. Regarding the Cronbach Alfa, the total factors of the CPDI scale and the Fear scale obtained the greatest internal consistencies, being 0.90 and 0.88 respectively.

Bartlett's sphericity test shows that the variables observed by the questionnaire are correlated in the population, Table 3.

Finally, the Kaiser-Meyer-Olkin (KMO) sample size suitability factor shows that the sample size is sufficiently adequate to test the variables for which they are intended by Factor Analysis, with all CPDI values being $\geq 0.82$ except question 21 (0.57) and all FEAR Scale values being $\geq 0.84$.

The CPDI scores of the comparison between the study groups, the Wilcoxon-Mann-Whitney test showed that people with psychological problems or who reported psychological suffering and heart disease and/or hypertension had the highest scores in the comparison with people who reported no health problems (Mann-Whitney $U=6399,5 ; \mathrm{p}<0.05$ ), and an important finding is the highest score of hypertensive people compared to all other groups, with higher scores even than those with psychological problems or who reported psychological suffering. In the assessment of volunteers who had diabetes, no statistically significant difference was found in comparison with the group of people without health problems. (Mann-Whitney $\mathrm{U}=42672 ; \mathrm{p}=0.88$ ). These data are shown in Table 4.

With regard to CPDI, the question with the highest score was question 8 , "I believe in Covid-19 information from all sources without any evaluation", which reinforces the influence of the information in the results of this research. Another point worth mentioning was the score for question 5, "I feel sympathy for the patients at Covid-19 and their families", which obtained the second-highest

Table 2. Cronbach's alpha: means and confidence interval by a factor-CPDI and fear scales.

\begin{tabular}{|c|c|c|c|c|c|c|c|c|}
\hline Class & Minimum & Maximum & Median & $\begin{array}{l}\text { Simple } \\
\text { Average }\end{array}$ & $\begin{array}{l}\text { Weighted } \\
\text { average }\end{array}$ & $\begin{array}{c}\text { Confidence Interval } \\
(95 \%)\end{array}$ & $\begin{array}{l}\text { Standard } \\
\text { deviation }\end{array}$ & $\begin{array}{c}\text { Cronbach's } \\
\text { alpha }\end{array}$ \\
\hline CPDI Fator 1 & 0.00 & 4.00 & 2.00 & 1.99 & 1.99 & $1.95-2.03$ & 1.0 & 0.74 \\
\hline CPDI Fator 2 & 0.00 & 3.57 & 1.14 & 1.16 & 1.17 & $114-1.20$ & 0.61 & 0.63 \\
\hline CPDI Fator 3 & 0.00 & 4.00 & 1.86 & 1.90 & 1.87 & $1.85-1.94$ & 0.92 & 0.83 \\
\hline Total CPDI & 4.00 & 93.00 & 38.00 & 39.57 & 39.57 & $38.83-40.31$ & 16.24 & 0.90 \\
\hline Fear Score & 7.00 & 35.00 & 17.00 & 18.08 & 1.99 & $17.78-18.39$ & 6.69 & 0.88 \\
\hline
\end{tabular}

Table 3. Bartlett's test-CPDI and fear scales.

\begin{tabular}{cccc}
\hline Class & Estimate & Degrees of freedom & p-value \\
\hline CPDI Scale & 2135.7 & 23 & 0.00 \\
Fear Scale & 466.42 & 6 & 0.00 \\
\hline
\end{tabular}


score among the volunteers, in this group, question 1, "Compared to the usual, I feel more nervous and anxious "obtained the third-highest score, already in the assessment of diabetics, the highest score was found in question 1, "Compared to the usual, I feel more nervous and anxious". Among hypertensive patients, there was a strong influence of question 1, showing high levels of nervousness anxiety, it is worth highlighting the high score concerning the question 6, "I feel powerless and angry with the people around me, such as the government and media". A point to be highlighted was the high score in question 14, "I feel tired and sometimes exhausted".

In Table 5, is referring to the FEAR scale scores. The G3 had the FCV-19S higher in comparison with G1, G2 and G4. The Wilcoxon-Mann-Whitney test showed that the comparison between the study groups shows behaviour similar to the data in Table 4 referring to the CPDI scale (Mann-Whitney $\mathrm{p}<0.05$ ), except in the comparisons between diabetics and without health problems, where although there is no statistically significant difference, as found in the CPDI, there was a tendency to have a greater impact on diabetic patients Mann-Whitney $\mathrm{U}=$ 47,572; $\mathrm{p}<0.07)$.

In FEAR scale, Table 5, the question with the highest score among volunteers with previous psychological distress was question 1, "I am very afraid of Covid-19", and question 5 "When watching the news and stories about Covid-19 on social media, I get nervous or anxious ", Showing that the information can positively or negatively influence the psychological condition during the pandemic,

Table 4. CPDI Score-Comparison between groups.

\begin{tabular}{|c|c|c|c|c|c|c|c|c|c|}
\hline Variable & Class & Media & $\begin{array}{l}\text { Media Confidence } \\
\text { Interval (IC)-95\% }\end{array}$ & $\begin{array}{l}\text { Minimum } \\
\text { Value }\end{array}$ & $1^{\text {st }}$ quartile & Median & $3^{\text {rd }}$ quartile & $\begin{array}{l}\text { Maximum } \\
\text { Value }\end{array}$ & p-valor \\
\hline \multirow[t]{2}{*}{ CPDI Score } & Diabetics & 37.93 & $34.05-41.80$ & 10.00 & 25.00 & 35.00 & 47.00 & 91.00 & 0.88 \\
\hline & No health problems & 37.33 & $36.49-38.18$ & 4.00 & 25.00 & 36.00 & 48.00 & 85.00 & \\
\hline \multirow[t]{2}{*}{ CPDI Score } & Hypertensive/Cardiac Diseases & 58.89 & $51.10-66.68$ & 19.00 & 50.00 & 61.00 & 70.50 & 93.00 & 0.00 \\
\hline & No health problems & 37.33 & $36.49-38.18$ & 4.00 & 25.00 & 36.00 & 48.00 & 85.00 & \\
\hline \multirow[t]{2}{*}{ CPDI Score } & With psychological distress & 51.62 & $49.87-53.44$ & 11.00 & 41.75 & 54.00 & 62.00 & 93.00 & 0.00 \\
\hline & No psychological distress & 37.28 & $36.52-38.05$ & 4.00 & 25.00 & 36.00 & 47.00 & 92.00 & \\
\hline
\end{tabular}

Table 5. Fear Score-Comparison between groups.

\begin{tabular}{|c|c|c|c|c|c|c|c|c|c|}
\hline Variable & Class & Media & $\begin{array}{c}\text { Media Confidence } \\
\text { Interval (IC)-95\% }\end{array}$ & $\begin{array}{l}\text { Minimum } \\
\text { Value }\end{array}$ & $1^{\text {st }}$ quartile & Median & $3^{\text {rd }}$ quartile & $\begin{array}{c}\text { Maximum } \\
\text { Value }\end{array}$ & p-valor \\
\hline \multirow{2}{*}{ Fear Score } & Diabetics & 18.90 & $17.19-20.61$ & 7.00 & 13.00 & 18.00 & 23.75 & 35.00 & 0.07 \\
\hline & No health problems & 17.28 & $16.92-17.64$ & 7.00 & 12.00 & 16.00 & 22.00 & 35.00 & \\
\hline \multirow{2}{*}{ Fear Score } & Hypertensive/Cardiac Diseases & 23.78 & $20.86-26.69$ & 7.00 & 19.00 & 25.00 & 29.50 & 34.00 & 0.00 \\
\hline & No health problems & 17.28 & $16.92-17.64$ & 7.00 & 12.00 & 16.00 & 22.00 & 35.00 & \\
\hline \multirow{2}{*}{ Fear Score } & With psychological distress & 20.84 & $20.06-21.62$ & 7.00 & 15.00 & 21.00 & 26.00 & 35.00 & 0.00 \\
\hline & No psychological distress & 17.56 & $17.23-17.89$ & 7.00 & 13.00 & 17.00 & 22.00 & 35.00 & \\
\hline
\end{tabular}


for diabetics, question 1 was also the one with the highest score, question 5 also showed strong influence, in this group, question 4, "I am afraid of losing my life because of COVID-19" presented the second-highest score. Hypertensive patients, on the other hand, had the highest scores in question 1, and 4, showing a strong influence of fear in this group, which is repeated with the influence of the news about the pandemic, demonstrated in question 6 , "when watching the news about COVID-19 on social media I get nervous or anxious".

\section{Discussion}

Psychiatric disorders and impairments of the psychological reactions have been related to cardiovascular diseases, especially hypertension, which is also observed in diabetic patients. Moreover, in a pandemic situation, little is known about the impacts of an event of the magnitude of COVID-19 pandemic on the mental health of hypertensive and diabetic patients. Studies including systematic reviews have shown that people with chronic diseases are more impacted by COVID-19 in comparison with healthy people, then the risk of contracting the severe form of Sars-CoV-2 [11] [12], and the psychological consequences of the pandemic by COVID-19, but specific data on patients with cardiovascular diseases and diabetes are scarce [7].

In response to the outbreak of the novel Severe Acute Respiratory Syndrome Coronavirus-2, a pathogen of the new coronavirus disease, several sectors and social activities have been affected, including education. At first, it is explained that educators and students can feel fragile during and after the Sars-CoV-2 outbreak. Subsequently, it is discussed that their relationship ought to be carefully established given the triggering of psychological and neuropsychiatric effects arising from neural coding and plasticity processes, which result in the formation of positive and negative memories in the short to long term [13].

Moreover, the population exposure to trauma, such as witnessing and caring for severely ill people, perceived life-threatening, mortality and bereavement, deaths of health professionals may impair the population's mental health, increasing the risks of developing psychic suffering and progression to psychopathology, among them post-traumatic stress disorder (PSTD) [14] [15].

People react to stressful psychological factors such as fear, indifference, fatalism, anxiety, depression and the like very differently from each other [16]. Besides, hypertension, diabetes and cardiovascular diseases have been identified as possible risk factors for more severe in patients with Sars-CoV-2, this may lead this part of the population to present higher levels of psychological symptoms since COVID-19 tends to present more severely in individuals with multiple comorbidities [17].

Furthermore, significant gender differences as in both scales women's mean scores are higher showing that it is paramount that women's voices were represented in policy spaces as socially constructed gender roles place them in a strategic position to enhance multi-level interventions (primary and secondary 
effects of COVID-19), equitable policies, and new approaches to control the pandemic [1] [4].

Wang and collaborators (2020), evaluated 1210 volunteers through the Depression, Anxiety and Stress Scale (DASS-21). In total, $53.8 \%$ of the interviewees classified the psychological impact of the outbreak as moderate or severe; $16.5 \%$ reported moderate to severe depressive symptoms; $28.8 \%$ reported moderate to severe anxiety symptoms, and $8.1 \%$ reported moderate to severe stress levels [18].

Recent studies have also shown that this increased anxiety resulting from COVID-19 could be particularly prevalent among people with a history of psychiatric problems. Moreover, some researchers have also pointed out that people with chronic diseases are expected to have higher levels of psychological symptoms since COVID-19 tends to be more severely manifest in those people with multiple underlying diseases [18]. Furthermore, to deal with and eliminate the stigma associated with the epidemic, it is necessary to create strategies to deal with the psychological stress of the post-pandemic period [3], especially people with chronic diseases.

In our study we found a high level of psychological suffering in patients with cardiovascular and diabetic diseases, which can be explained in part by the high levels of psychological suffering found in hypertensive and diabetic patients even at non-pandemic times, however, there is evidence of a greater impact of the pandemic by COVID-19 on the mental health of this part of the population.

In a study of 52,095 hypertensive patients from 19 countries, Stein et al., 2014 describe that depression, anxiety and eating disorders were significantly associated with the subsequent diagnosis of hypertension [19]. Patients with a lifetime anxiety disorder reported higher rates of several medical illnesses than did persons without anxiety. After controlling for the effects of gender, comorbid substance abuse/dependence and/or depression, significant associations were found between anxiety disorder and cardiac disorders $(\mathrm{OR}=4.6)$, hypertension $(\mathrm{OR}=2.4)$, gastrointestinal problems $(\mathrm{OR}=2.4)$, genitourinary disorders $(\mathrm{OR}=$ $3.5)$, and migraine $(\mathrm{OR}=5.0)$. A similar pattern was observed for probands with panic or generalized anxiety disorder (GAD) [20].

Li et al., 2015 in a meta-analysis of 10,194 hypertensive patients in 27 studies using self-evaluation scales of depression found a high prevalence of depression (29.8\%), similar numbers to those found by Gebre et al., 2020 in a study of 310 hypertensive patients (24.7\%) [21] [22].

Diabetic patients, on the other hand, present similar levels of psychological suffering, ranging from $17 \%$ to $28.8 \%$ in different studies and countries [23] [24]. In our study, people with diabetes had high levels of stress and fear, but with no statistical difference to volunteers without diabetes, however, both have high levels of stress and fear in the face of the pandemic by COVID-19.

Studies show that individuals with severe illness or multiple comorbidities present higher levels of psychological symptoms in a crisis. In a study that assessed 
the impact of the COVID-19 pandemic in Spain, Ozamiz-Etxebarria et al., 2020, showed that individuals who reported chronic diseases had higher average levels of stress, anxiety and depression compared to participants who did not report such diseases [8].

Li et al., 2020, in a study that evaluated 1099 patients with COVID-19, of which 173 had the severe form of the disease having as risk factors comorbidities, being arterial hypertension (23.7\%), diabetes mellitus (16.2\%), coronary diseases (5.8\%) and cerebrovascular disease (2.3\%) [7].

Recent theories regarding the etiology of depression have involved the biogenic amine pathway, and suggest that the disease is related to a deficiency in monoamines (serotonin, dopamine and norepinephrine) in the central nervous system. Indeed, all clinical antidepressant drugs enhance the effects of monoamine neurotransmitters. Both depressive and hypertensive patients experience increased sympathetic tone and increased secretion of adrenocorticotropic hormone and cortisol; therefore, it is pathophysiologically plausible that depression and hypertension affect one another [25].

In fact, in our study, individuals with cardiovascular diseases, including hypertension, had significantly higher levels of peri-traumatic fear and stress than individuals who do not have chronic diseases. The scores assessed by the Fear scale (FCV-19S) were 23.78, indicating a mean level of fear, which is repeated with the evaluation of peri-traumatic stress by the Peri-Traumatic Distress Index (CPDI) with a score of 58.89 indicating a severe score. This shows that this population should receive special attention from public health agencies with the implementation of policies aimed at mental health monitoring during the pandemic and post-pandemic periods. The self-efficacy of human beings seems to strongly rely on the stability of the notion that based on our yesterday's experience we trust that we can strongly rely on our today's condition and predict the environmental changes of tomorrow [26]. For that, it is necessary to identify the most vulnerable, promote risk management and implement public health measures aimed at-risk groups.

At last, it is relevant to mention that this study has some limitations. First, although the participants were people for any region of Brazil, this survey should not be taken as a national sample; secondly, as most of the respondents had an incomplete or complete college degree, it does not reflect most of the Brazilian population; third, data on diseases are self-reported by volunteers.

\section{Conclusion}

In conclusion, it was evidenced that two main factors lead hypertensive and diabetic patients to have such high levels of psychic suffering during the pandemic by COVID-19, first that this population already suffers from higher levels of depression and other psychic problems, according to the knowledge and constant disclosure that these people belong to the group at risk for development of severe form and evolution to death by COVID-19. 


\section{Acknowledgements}

We acknowledge the participants in this study.

\section{Authors' Contribution}

L.E.P.P. Teixeira, A. Abad, Juliana A. da Silva and R.L. de Freitas elaborated, analysed, and wrote sed the manuscript; José A. da Silva participated in the created, elaborated the project and revised the manuscript; S. Paiva, S. Bastos, C.H.C. Mármora, L.A.M. Campos and M. Antonelli-Ponti, revised the manuscript.

\section{Conflicts of Interest}

The authors declare no conflicts of interest regarding the publication of this paper.

\section{References}

[1] Abad, A., da Silva, J.A., das Neves Braga, J.V.Z., Medeiros, P., de Freitas, R.L., Coimbra, N.C. and da Silva, J.A. (2020) Preparing for the COVID-19 Mental Health Crisis in Latin America-Using Early Evidence from Countries That Experienced COVID-19 First. Advances in Infectious Diseases, 10, 40-44. https://doi.org/10.4236/aid.2020.103005

[2] Danese, S., Sands, B., Ng, S.C. and Peyrin-Biroulet, L. (2020) The Day after COVID19 in IBD: How to Go Back to 'Normal'. Nature Reviews Gastroenterology \& Hepatology, 17, 441-443. https://doi.org/10.1038/s41575-020-0322-8

[3] Wang, C.Y., Pan, R.Y., Wan, X.Y., Tan, Y.L., Xu, L.K., Ho, C.S. and Ho, R.C. (2020) Immediate Psychological Responses and Associated Factors during the Initial Stage of the 2019 Coronavirus Disease (COVID-19) Epidemic among the General Population in China. International Journal of Environmental Research and Public Health, 17, 1729. https://doi.org/10.3390/ijerph17051729

[4] Abad, A., da Silva, J.A., de Paiva Teixeira, L.E.P., Antonelli-Ponti, M., Bastos, S., Mármora, C.H.C., Campos, L.A.M., Paiva, S., de Freitas, R.L. and da Silva, J.A. (2020) Evaluation of Fear and Peritraumatic Distress during COVID-19 Pandemic in Brazil. Advances in Infectious Diseases, 10, 184-194. https://doi.org/10.4236/aid.2020.103019

[5] Kang, Y., Chen, T., Mui, D., Ferrari, V., Jagasia, D., Scherrer-Crosbie, M., Chen, Y.C. and Han, Y.C. (2020) Cardiovascular Manifestations and Treatment Considerations in COVID-19. Heart, 106, 1132-1141. https://doi.org/10.1136/heartjnl-2020-317056

[6] Nasser, F.J., de Almeida, M.M., da Silva, L.S., de Almeida, R.G.P., Barbirato, G.B., Mendlowicz, M.V. and Mesquita, C.T. (2016) Psychiatric Disorders and Cardiovascular System: Heart-Brain Interaction. International Journal of Cardiovascular Sciences, 29, 65-75. https://doi.org/10.5935/2359-4802.20160003

[7] Li, B., Yang, J., Zhao, F.M., Zhi, L.L., Wang, X.Q., Liu, L., Bi, Z.H. and Zhao, Y.H. (2020) Prevalence and Impact of Cardiovascular Metabolic Diseases on COVID-19 in China. Clinical Research in Cardiology, 109, 531-538. https://doi.org/10.1007/s00392-020-01626-9

[8] Ozamiz-Etxebarria, N., Dosil-Santamaria, M., Picaza-Gorrochategui, M. and Idoiaga-Mondragon, N. (2020) Stress, Anxiety, and Depression Levels in the Initial Stage 
of the COVID-19 Outbreak in a Population Sample in the Northern Spain. Cadernos de Saúde Pública, 36, e00054020. https://doi.org/10.1590/0102-311x00054020

[9] Qiu, J.Y., Shen, B., Zhao, M., Wang, Z., Xie, B. and Xu, Y.F. (2020) A Nationwide Survey of Psychological Distress among Chinese people in the COVID-19 Epidemic: Implications and Policy Recommendations. General psychiatry, 33, e100213. https://doi.org/10.1136/gpsych-2020-100213

[10] Ahorsu, D.K., Lin, C.Y., Imani, V., Saffari, M., Griffiths, M.D. and Pakpour, A.H. (2020) The Fear of COVID-19 Scale: Development and Initial Validation. International Journal of Mental Health and Addiction. https://doi.org/10.1007/s11469-020-00270-8

[11] Wang, X.H., Fang, X.X., Cai, Z.X., Wu, X.T, Gao, X.T, Min, J.X. and Wang, F.D. (2020) Comorbid Chronic Diseases and Acute Organ Injuries Are Strongly Correlated with Disease Severity and Mortality among COVID-19 Patients: A Systemic Review and Meta-Analysis. Research, 2020, Article ID: 2402961. https://doi.org/10.34133/2020/2402961

[12] Liu, H., Chen, S.y., Liu, M., Nie, H. and Lu, H.Y. (2020) Comorbid Chronic Diseases are Strongly Correlated with Disease Severity among COVID-19 Patients: A Systematic Review and Meta-Analysis. Aging and Disease, 11, 668-678.

https://doi.org/10.14336/AD.2020.0502

[13] Naves-Barcelos, M., Medeiros, P., da Sliva Tostes, C.B., da Silva, J.A., Torres da Silva, J.R.T., Tostes, J.G., da Silva, J.A., Coimbra, N.C., da Silva, M.L., de Freitas, R.L. (2020) Role of Education after the COVID-19 Pandemic Fear: A Multidisciplinary and Scientific Perspective. SciELO Preprints. https://doi.org/10.1590/SciELOPreprints.710

[14] Shultz, J.M., Baingana, F. and Neria, Y. (2015) The 2014 Ebola Outbreak and Mental Health: Current Status and Recommended Response. JAMA, 313, 567-568. https://doi.org/10.1001/jama.2014.17934

[15] Neria, Y. and Sullivan, G.M. (2011) Understanding the Mental Health Effects of Indirect Exposure to Mass Trauma through the Media. JAMA, 306, 1374-1375. https://doi.org/10.1001/jama.2011.1358

[16] Taylor, S. (2019) The Psychology of Pandemics: Preparing for the Next Global Outbreak of Infectious Disease. Cambridge Scholars Publishing, Newcastle.

[17] Martinez-Ferran, M., de la Guía-Galipienso, F., Sanchis-Gomar, F. and Pareja-Galeano, H. (2020) Metabolic Impacts of Confinement during the COVID-19 Pandemic Due to Modified Diet and Physical Activity Habits. Nutrients, 12, 1549. https://doi.org/10.3390/nu12061549

[18] Ozamiz-Etxebarria, N., Idoiaga Mondragon, N., Dosil Santamaría, M. and Picaza Gorrotxategi, M. (2020) Corrigendum: Psychological Symptoms during the Two Stages of Lockdown in Response to the COVID-19 Outbreak: An Investigation in a Sample of Citizens in Northern Spain. Frontiers in Psychology, 11, 1491. https://doi.org/10.3389/fpsyg.2020.02116

[19] Stein, D.J., Aguilar-Gaxiola, S., Alonso, J., Bruffaerts, R., de Jonge, P., Liu, Z., Miguel Caldas-de-Almeida, J., O’Neill, S., Viana, M.C., Al-Hamzawi, A.O., Angermeyer, M.C., Benjet, C., de Graaf, R., Ferry, F., Kovess-Masfety, V., Levinson, D., de Girolamo, G., Florescu, S., Hu, C., Kawakami, N., Scott, K.M., et al. (2014) Associations between Mental Disorders and Subsequent Onset of Hypertension. General Hospital Psychiatry, 36, 142-149.

https://doi.org/10.1016/j.genhosppsych.2013.11.002

[20] Härter, M.C., Conway, K.P. and Merikangas, K.R. (2003) Associations between An- 
xiety Disorders and Physical Illness. European Archives of Psychiatry and Clinical Neurosciences, 253, 313-320. https://doi.org/10.1007/s00406-003-0449-y

[21] Li, Z.Z., Li, Y.Y., Chen, L.Z., Chen, P. and Hu, Y.Y. (2015) Prevalence of Depression in Patients with Hypertension: A Systematic Review and Meta-Analysis. Medicine (Baltimore), 94, e1317.

[22] Gebre, B.B., Deribe, B. and Abeto, M. (2020) Magnitude and Associated Factors of Depression Among Hypertensive Patients Attending Treatment Follow Up in Chronic OPD at Hawassa University Comprehensive Specialized Hospital, Hawassa, Southern Ethiopia. Integrated Blood Pressure Control, 2020, 31-39. https://doi.org/10.2147/IBPC.S240015

[23] Park, C.Y., Kim, S.Y., Gil, J.W., Park, M.H., Park, J.H. and Kim, Y.J. (2015) Depression among Korean Adults with Type 2 Diabetes Mellitus: Ansan-CommunityBased Epidemiological Study. Osong Public Health and Research Perspectives, 6 , 224-232. https://doi.org/10.1016/j.phrp.2015.05.004

[24] Alajmani, D.S.A., Alkaabi, A.M., Alhosani, M.W., Folad, A.A., Abdouli, F.A., Carrick, F.R. and Abdulrahman, M. (2019) Prevalence of Undiagnosed Depression in Patients with Type 2 Diabetes. Frontiers in Endocrinology, 10, 259.

https://doi.org/10.3389/fendo.2019.00259

[25] Rubio-Guerra, A.F., Rodriguez-Lopez, L., Vargas-Ayala, G., Huerta-Ramirez, S., Serna, D.C. and Lozano-Nuevo, J.J. (2013) Depression Increases the Risk for Uncontrolled Hypertension. Experimental and Clinical Cardiology, 18, 10-12.

[26] Kreuzer, P.M., Baghai, T.C., Rupprecht, R., Wittmann, M., Steffling, D., Ziereis, M., Zowe, M., Hausner, H. and Langguth, B. (2020) SARS-CoV-2 Risk Management in Clinical Psychiatry: A Few Considerations on How to Deal with an Unrivaled Threat. Frontiers in Psychiatry, 11, 550. https://doi.org/10.3389/fpsyt.2020.00550 


\section{Appendices}

\section{Appendix 1: Peri-Traumatic Distress Scale (CPDI)—COVID-19}

1) Compared to usual, I feel more nervous and anxious.

2) I feel insecure and bought a lot of masks, medications, sanitizer, gloves and/or other home supplies.

3) I can't stop myself from imagining myself or my family being infected and feel terrified and anxious about it.

4) I feel empty and helpless no matter what I do.

5) I feel sympathetic to the COVID-19 patients and their families. I feel sad about them.

6) I feel helpless and angry about people around me, governors, and media.

7) I am losing faith in the people around me.

8) I collect information about COVID-19 all day. Even if it's not necessary, I can't stop myself.

9) I will believe the COVID-19 information from all sources without any evaluation.

10) I would rather believe in negative news about COVID-19 and be skeptical about the good news.

11) I am constantly sharing news about COVID-19 (mostly negative news).

12) I avoid watching COVID-19 news, since I am too scared to do so.

13) I am more irritable and have frequent conflicts with my family.

14) I feel tired and sometimes even exhausted.

15) I find it hard to concentrate.

16) I find it hard to make any decisions.

17) During this COVID-19 period, I often feel dizzy or have back pain and chest distress.

18) During this COVID-19 period, I often feel dizzy or have back pain and chest distress.

19) During this COVID-19 period, I often feel stomach pain, bloating, and other stomach discomfort.

20) I feel uncomfortable when communicating with others.

21) Recently, I rarely talk to my family.

22) I cannot sleep well. I always dream about myself or my family being infected by COVID-19.

23) I lost my appetite.

24) I have constipation or frequent urination.

\section{Appendix 2: Fear of COVID-19 Scale (Ahorsu et al., 2020)}

1) I am most afraid of coronavirus-19.

2) It makes me uncomfortable to think about the coronavirus-19.

3) My hands become clammy when I think about coronavirus-19.

4) I am afraid of losing my life because of coronavirus-19.

5) When watching news and stories about coronavirus-19 on social media, I 
become nervous and anxious.

6) I cannot sleep because I'm worrying about getting coronavirus-19

7) My heart races or palpitates when I think about getting coronavirus-19. 